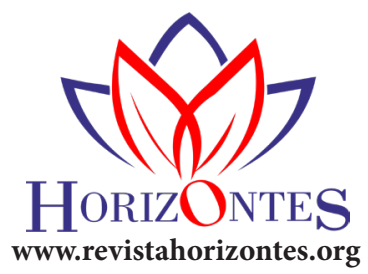

Horizontes. Revista de Investigación en Ciencias de la Educación Https://doi.org/10.33996/revistahorizontes.v5i21.307

Octubre-diciembre 2021

Volumen 5 / No. 21

ISSN: 2616-7964

ISSN-L: 2616-7964

pp. 1673 - 1682

\title{
Estrategias metodológicas en proceso de enseñanza y aprendizaje de la gimnasia para estudiantes de Educación Física
}

\author{
Methodological strategies in the teaching process - gymnastics learning for physical education students
}

Estratégias metodológicas no processo de ensino - aprendizagem de ginástica para alunos de Educação Física

\section{RESUMEN}

El articulo tuvo como objetivo "evaluar las estrategias metodológicas", con el fin de conocer e indagar cuáles son las aplicadas en el proceso de enseñanza aprendizaje de la gimnasia de Educación Física de La Escuela Profesional de Educación (UNSAAC) para el año 2018. La investigación fue de tipo "aplicada"; con un nivel "descriptivo". En tal sentido, se seleccionó una muestra de 97 participantes entre docentes especialistas y estudiantes cursantes de la especialidad de Educación Física para la aplicación de un cuestionario de 20 ítems para conocer su perspectiva del estudio. Se concluyó que, las estrategias metodológicas aplicadas en el proceso de enseñanza-aprendizaje de la UNSAAC en la especialidad de Educación Física, son eficientes.

Palabras clave: Educación Física, Gimnasia; Docentes; Estudiantes; Universidad
ABSTRACT

The objective of the article was to "evaluate the methodological strategies", in order to know and investigate which ones are applied in the teachinglearning process of Physical Education gymnastics of the Professional School of Education (UNSAAC) for the year 2018. The research was of the "applied" type; with a "descriptive" level and a "qualitative" approach to understand the proposed study phenomenon. In this sense, a sample of 97 participants was selected among specialist teachers and students studying the specialty of physical education for the application of a 20-item questionnaire to know their perspective of the study. It was concluded that the methodological strategies applied in the teaching-learning process of the UNSAAC in the specialty of physical education, are efficient.

Key words: Physical education; Gymnastics; Teacher; Students; University

\section{RESUMO}

$\mathrm{O}$ artigo teve como objetivo "avaliar as estratégias metodológicas", a fim de conhecer e investigar quais são aplicadas no processo de ensino-aprendizagem da ginástica laboral da Educação Física da Escola Profissional de Educação (UNSAAC) para o ano de 2018. A pesquisa foi do tipo "aplicado"; com um nível "descritivo" e uma abordagem "qualitativa" para a compreensão do fenômeno de estudo proposto. Nesse sentido, uma amostra de 97 participantes foi selecionada entre professores especialistas e alunos da especialidade educação física para a aplicação de um questionário de 20 itens para conhecer a perspectiva do estudo. Concluiu-se que as estratégias metodológicas aplicadas no processo de ensino-aprendizagem da UNSAAC, na especialidade de Educação Física, são eficazes.

Palavras-chave: Educação física; Ginástica; Professore; Alunos; Universidade 


\section{INTRODUCCIÓN}

En Europa, a principios del siglo XIX, comenzó a practicarse la gimnasia y se conocía como "ejercicios físicos" con o sin aparatos, también, como "gimnasia en grandes aparatos"; considerándose como precursor Johann Friedrich-LudwigChristoph Jahn ya que expandió dicha práctica por Alemania (Prusia, 1778, Freyburg, 1825) (Cachorro y Giles, 2019).

Desde el inicio hasta la actualidad, hubo un proceso de transformación en la práctica ya que la interpretación de los movimientos ha sido cada vez más estilizados y con mayor detalle en la expresión (Cachorro y Giles, 2019).

Barragán (2018) indica que, en el caso de la práctica de la gimnasia, pone en desarrollo las habilidades y destrezas en los niños y niñas de edad primaria, a su vez, eleva su nivel educativo y psicológico. Además, Hinostroza (2019) indica que, la organización y el tratamiento adecuado del contenido de aprendizaje, a su vez, el uso de estrategias metodológicas adecuadas y variadas, promueven las interacciones significativas entre el docente, los estudiantes y material educativo suministrado. Asimismo, Reguena (2018) propone que, la intervención didáctica gimnástica eficaz mejora de la conducta socialmente hábil de los adolescentes. Por su parte, favorece el aprendizaje de los jóvenes para trabajar en grupo y aceptación de los compañeros.

Sin embargo, la poca utilización de estrategias metodológicas para el desarrollo del gimnasta no permite el desarrollo de sus propias habilidades y destrezas, cognitivas y motoras mediante la gimnasia. Por ello, la enseñanza de habilidades gimnasticas requiere de programas y/o estrategias de enseñanza; que involucre a los gimnastas en conductas motrices concretas en sus propósitos, siendo necesaria e imprescindible la manipulación intencionada de los ambientes de aprendizaje, mediante la planificación de espacios, recursos $\mathrm{y}$ materiales que promuevan su experiencia deportiva y personal (Mamani, Laque y Mamani, 2019). En consecuencia, Huamani (2019) afirma la importancia de la práctica de la gimnasia rítmica favorece el desarrollo psicomotor de los niños y hasta los jóvenes, a su vez, contribuye su formación integral.

Cabe destacar que, Pinela (2016) indica que el especialista selecciona las estrategias y técnicas más acorde para el desarrollo de cada estudiante. Así como, Estupiñán y Pérez (2015) plantea trabajar los elementos rítmicos -pulso, acento, ritmo- y el silencio musical; trabajar los movimientos largos y cortos con precisión; permite prestar la atención detenida los sonidos y producir sonidos con su cuerpo, objetos y con instrumentos musicales. En efecto, Villalobos (2015) afirma que se debe crear contra con una infraestructura acorde para el deporte multidisciplinario, es decir, aquella que opten las condiciones adecuadas de acuerdo a las funciones de espacio, forma, concepto y función de la misma para satisfacer las necesidades de la población deportista.

Por su parte, Ticona (2017) recomienda a potencializar la enseñanza y aprendizaje de la gimnasia deportiva desde la escolaridad temprana. Del mismo modo, Blas y Díaz (2018), la gimnasia es fundamental para niños de 3 años ya que permite desarrollar la motricidad gruesa. Sánchez (2019), la práctica de la Gimnasia Sueca favorece el desarrollo de la coordinación motora gruesa, pero se debe considerar el desarrollo individual de cada niño. 
Por otro lado, Guzmán (2019) opina que, el docente de Educación Física no motiva para mejorar sus capacidades y habilidades como estudiante. En consecuencia, el bajo conocimiento de las disciplinas deportivas, el poco fomento de valores como el respeto y el compañerismo y la poca motivación de los estudiantes ha evidenciado la ausencia de estrategias y metodologías del docente de Educación Física en el proceso de enseñanzaaprendizaje.

Esta situación esta determinada por la poca formación del recurso humano en las diversas estrategias metodológicas en el proceso de enseñanza de la gimnasia, lo cual produce un bajo rendimiento en los estudiantes que la cursan. Es importante desarrollar estrategias que vayan de la mano con los contenidos del deporte. Se pueden aplicar estrategias mixtas como el conductivismo, dedica a los docentes a explicar directamente los movimientos de gimnasia a los estudiantes, el constructivismo donde los jóvenes investigan, aprende y exponen sus experiencias en pro del desarrollo de nuevos movimientos y el conectivismo que entrelaza esos nuevos conocimientos a través de la aplicación de las Tecnologías de la Información y Comunicación (TIC), donde hace aún más protagonista de su desarrollo a los estudiantes.

Los métodos pedagógicos son importantes en cualquier área de las ciencias. Para el caso de la Educación Física, permiten desarrollar nuevas estrategias de enseñanza con el fin de generar los conocimientos necesarios para el desarrollo de la gimnasia en los estudiantes. Los docentes deben hacer procesos sinérgicos con actitudes motivacionales que impulsen las técnicas deportivas en los estudiantes. La motivación, compromiso, responsabilidad y los conocimientos permitirán desarrollar las actividades físicas con éxitos, enmarcadas en altas tasas de calidad.

Por otra parte, a nivel artístico, la gimnasia se divide dos ramas, la primera denominada "Gimnasia Artística Femenina" y la segunda, “Gimnasia Artística Masculina”. En tal sentido, los aparatos correspondientes al estilo femenino son: Salto, Viga, Paralelas asimétricas y Suelo (Orden de rotación Olímpico). En cambio, los aparatos correspondientes al estilo masculino son: Suelo, Caballo con Arzones, Anillas, Salto, Paralelas y Barra Fija. (Orden de rotación Olímpico). Cabe destacar que, el Suelo y el Salto se comparten en ambos estilos, sin embargo, las obligaciones y exigencias son diferentes para ambas ramas (Cachorro y Giles, 2019).

En Perú, de acuerdo con la Federación Deportiva Peruana de Gimnasia, surge a partir del año 2013, el Club Escuela de Gimnasia de Lima como institución constituida para la formación de la disciplina, es decir, dicha práctica gimnástica es reciente y muy limitado, sobre todo, en las regiones más lejanas de la capital. Sin embargo, existe una Federación Deportiva Peruana de Gimnasia adscrita a Instituto Peruano del Deporte considerado como el ente rector del Sistema Deportivo Nacional. Dicha Federación cuenta con un Club para incentivar la práctica gimnastica.

En Cusco, específicamente, en la Universidad Nacional de San Antonio Abad del Cusco, la Facultad de Educación en la especialidad de Educación Física plantea en su plan de estudios sólo 2 cursos para la práctica de la gimnasia. Tanto los profesores como los estudiantes, han cuestionado el número de asignaturas vinculados con la gimnasia, la 
infraestructura para llevar a cabo dichas prácticas y en especial, cuáles son las estrategias metodológicas aplicadas para el proceso de enseñanza-aprendizaje de la misma. Así como, Mina y Andino (2017) indica que, se debe fomentar la revisión de estudios para elevar el conocimiento pedagógico.

En consecuencia, surge la siguiente necesidad: ¿Cuáles son las estrategias metodológicas en el proceso de enseñanza - aprendizaje de la gimnasia para los estudiantes de Educación Física de la Escuela Profesional de Educación de la Universidad Nacional De San Antonio Abad del Cusco, 2018? Además, conocer cuáles son las perspectivas tanto de los docentes especialistas como de los estudiantes cursantes de la misma.

\section{MÉTODO}

Se realizó un estudio aplicado para poder responder a las necesidades de las estrategias metodológicas para la enseñanza de la Educación Física aplicada a los estudiantes que las cursan. Del mismo modo, la investigación es de tipo descriptiva, asimismo, la investigación es de carácter exploratorio, también la investigación es de tipo explicativa.

Con respecto a, el diseño estadístico para probar la hipótesis utilizado fue la "media aritmética", considerando la hipótesis nula y alterna según la variable de estudio.
Ha: "Las estrategias metodológicas en el proceso de enseñanza y aprendizaje de la gimnasia para los estudiantes de Educación Física de la Escuela Profesional de Educación de la Universidad Nacional De San Antonio Abad Del Cusco, 2018 no son eficientes".

Ho: "Las estrategias metodológicas en el proceso de enseñanza - aprendizaje de la gimnasia para los estudiantes de Educación Física de la Escuela Profesional de Educación de la Universidad Nacional De San Antonio Abad Del Cusco, 2018 son eficientes".

Asimismo, la población estuvo conformada por 46 docentes y 148 estudiantes de la especialidad de Educación Física de la Escuela Profesional de Educación UNSAAC, Cusco para el año 2018. La muestra finita seleccionada fue de 97 personas representada por estudiantes y docentes. La fracción de la muestra es de 0,5 , por lo que la cantidad de estudiantes en la muestra es de 74 y de docentes es de 23.

Se aplicó como técnica e instrumento de recolección de datos la encuesta y el cuestionario. Estos buscan reflejar la percepción de los docentes y estudiantes con respecto a las metodologías aplicadas en la enseñanza de Educación Física. Las respuestas se basaron en escalamiento tipo Likert. La encuesta se detalla en la Tabla 1.

Tabla 1. Encuesta aplicada a la muestra estudio de la Escuela Profesional de Educación UNSAAC.

\begin{tabular}{|c|c|c|c|c|c|c|}
\hline \multirow{2}{*}{$\mathbf{N}^{\circ}$} & \multirow{2}{*}{ PREGUNTAS } & \multicolumn{5}{|c|}{ RESPUESTAS } \\
\hline & & 5 & 4 & 3 & 2 & 1 \\
\hline 1 & $\begin{array}{l}\text { Las estrategias metodológicas en la enseñanza de la gimnasia son favorables para el } \\
\text { aprendizaje de las destrezas gimnásticas de los estudiantes de Educación Física de la } \\
\text { UNSAAC. }\end{array}$ & & & & & \\
\hline 2 & $\begin{array}{l}\text { Autoridades y profesores deben originar actividades gimnásticas, con estudiantes de } \\
\text { Educación Física, como driles, etc. }\end{array}$ & & & & & \\
\hline
\end{tabular}


3 La gimnasia básica debe ser considerada en el plan curricular de la escuela profesional de Educación Física de la UNSAAC, como un curso no cognoscitivo.

4 La escuela profesional de Educación Física de la UNSAAC, debe contar con la infraestructura adecuada y pertinente para la práctica de la gimnasia de los estudiantes de la especialidad de Educación Física para un eficiente aprendizaje de las destrezas.

5 Debe el actual gimnasio que tiene la escuela profesional de educación, ser implementada de acuerdo a la necesidad de cada asignatura de especialidad que desarrollan los estudiantes.

6 Debería formularse trabajos, con los estudiantes de la especialidad de Educación Física según requerimientos del MINEDU.

7 Las estrategias que son aplicadas en el programa de Educación Física son de gran utilidad para los estudiantes, ya que estos aprenden prontamente y estos las aplican con eficiencia.

8 Los estudiantes de la especialidad de Educación Física, se preparan de forma eficaz con las estrategias metodológicas de enseñanza de la gimnasia, que se les imparte.

9 Las estrategias metodológicas de enseñanza de la gimnasia son variadas y permiten mejorar las destrezas gimnásticas.

10 Los profesores deben dar facilidades para realizar actividades gimnásticas a los estudiantes para desarrollar sus capacidades.

11 Deben los estudiantes contar con los materiales e implementos necesarios para el eficiente y eficaz aprendizaje de la gimnasia.

12 La escuela profesional de educación, debe promover implementos necesarios para la práctica de la gimnasia básica u otra.

13 Se debe impartir conocimientos de los reglamentos de la gimnasia a los estudiantes de Educación Física, para su mejor desempeño.

14 La liga provincial debe efectuar convenios con la Facultad de educación y ciencias de la comunicación para su organización, dirección y control de actividades gimnásticas.

15 Debe contar la carrera profesional de educación con el apoyo de personal especializado en gimnasia para mejorar el aprendizaje de las habilidades gimnásticas en los estudiantes.

16 En su composición, el plan de estudio de la especialidad de Educación Física debe de aumentar las asignaturas en gimnasia.

17 Las autoridades de la escuela profesional de educación y de la especialidad de Educación Física deben implementar cursos o seminarios de gimnasia para un mejor conocimiento en el desarrollo de las habilidades gimnásticas en los estudiantes. 
$\mathbf{N}^{\circ}$

PREGUNTAS
5

18 Debe implementar la pasantía de profesores y estudiantes de Educación Física para un mejor conocimiento en el proceso de enseñanza-aprendizaje de la gimnasia y mejorar las habilidades gimnásticas de los participantes en otras universidades.

19 Se debe incidir con cursos, seminarios y conferencias sobre las estrategias metodológicas en la enseñanza de la gimnasia para una mejor preparación y enseñanza de los profesores de especialidad de Educación Física.

20 Se debe incentivar a los estudiantes de la especialidad de Educación Física a la práctica permanente de la gimnasia para prepararlos y mejorar sus destrezas gimnásticas para un mejor desenvolvimiento profesional y una alta calidad de vida.

\begin{tabular}{ccccc}
\hline Totalmente de & De Acuerdo & Ni de Acuerdo ni en & En Desacuerdo & Totalmente en \\
Acuerdo & 4 & Desacuerdo & 2 & Desacuerdo \\
5 & & 3 & & 1 \\
\hline
\end{tabular}

Cabe destacar que, la consideración como ética profesional que fortalece la investigación es a través de la confidencialidad de los datos utilizados para la misma. En síntesis, los mismos fueron empleados para fines investigativos, es decir, sólo es de carácter académico.

\section{Análisis de datos}

Los datos obtenidos del instrumento aplicado a los docentes y estudiantes se basaron en la escala tipo Likert la cual tuvo una ponderación por cada respuesta. Las mismas fueron tabuladas para obtener la opinión de la muestra estudio, la cual refleja los resultados sobre la percepción de los estudiantes y profesores sobre las metodologías aplicadas en la enseñanza de la gimnasia en la Educación Física.

\section{RESULTADOS Y DISCUSIÓN}

Los resultados de las encuestas se verán reflejadas a través del análisis de las pruebas de hipótesis.

\section{Perspectiva de los docentes de la Escuela Profesional de Educación UNSAAC}

De acuerdo a la perspectiva de los profesores especialistas, un 53\% de los encuestados están “Totalmente de acuerdo" con que las estrategias para la adquisición de conocimiento, los métodos instructivos y el desarrollo de las capacidades y habilidades en el proceso de enseñanza y aprendizaje de la gimnasia para los estudiantes de Educación Física de la Escuela Profesional de Educación de la Universidad Nacional de San Antonio Abad del Cusco para el año 2018 son eficientes (Ver Tabla 2). 
Tabla 2. Perspectiva de los profesores de la especialidad de Educación Física.

\begin{tabular}{lcc}
\hline \multicolumn{1}{c}{ Escala } & Frecuencia & Porcentaje \\
\hline Totalmente de acuerdo & 82 & $53 \%$ \\
De acuerdo & 40 & $26 \%$ \\
Ni de acuerdo ni en desacuerdo & 20 & $13 \%$ \\
En desacuerdo & 12 & $8 \%$ \\
Totalmente en desacuerdo & 1 & $1 \%$ \\
\hline Total & $\mathbf{1 5 5}$ & $\mathbf{1 0 0 \%}$ \\
\hline
\end{tabular}

Perspectiva de los estudiantes de la Escuela Profesional de Educación UNSAAC

Por su parte, de acuerdo a la perspectiva de los estudiantes cursantes en la especialidad de Educación Física, un $40 \%$ de los encuestados están "De acuerdo" con que las estrategias para la adquisición de conocimiento, los métodos instructivos y el desarrollo de las capacidades y habilidades en el proceso de enseñanza-aprendizaje de la gimnasia para los estudiantes de Educación Física de la Escuela Profesional de Educación de la Universidad Nacional de San Antonio Abad del Cusco para el año 2018 son eficientes (Ver Tabla 3).

Tabla 3. Perspectiva de los estudiantes de la especialidad de Educación Física.

\begin{tabular}{lcc}
\hline \multicolumn{1}{c}{ Escala } & Frecuencia & Porcentaje \\
\hline Totalmente de acuerdo & 637 & $35 \%$ \\
De acuerdo & 719 & $40 \%$ \\
Ni de acuerdo ni en desacuerdo & 368 & $20 \%$ \\
En desacuerdo & 69 & $4 \%$ \\
Totalmente en desacuerdo & 16 & $1 \%$ \\
\hline Total & $\mathbf{1 8 0 9}$ & $\mathbf{1 0 0 \%}$ \\
\hline
\end{tabular}

\section{Resultado en base a las hipótesis}

Con respecto a, el diseño estadístico para probar la hipótesis utilizado fue la "media aritmética", considerando la hipótesis nula y alterna según la variable de estudio.

Ha: "Las estrategias metodológicas en el proceso de enseñanza y aprendizaje de la gimnasia para los estudiantes de Educación Física de la Escuela Profesional de Educación de la
Universidad Nacional De San Antonio Abad Del Cusco, 2018 no son eficientes".

Ho: "Las estrategias metodológicas en el proceso de enseñanza y aprendizaje de la gimnasia para los estudiantes de Educación Física de la Escuela Profesional de Educación de la Universidad Nacional De San Antonio Abad Del Cusco, 2018 son eficientes". 


$$
\bar{x}=\frac{\sum f i * X i}{n}
$$

Es decir, la media aritmética es 2,57.

Como un 2,57 de media, un nivel de confianza de $99 \%$ y un margen de error de $10 \%$; se establece que la enseñanza-aprendizaje de la gimnasia para los estudiantes de Educación Física de la Escuela Profesional de Educación de la Universidad Nacional De San Antonio Abad del Cusco para el año 2018 en la cual se acepta la Hipótesis Nula y se rechaza la Hipótesis Alterna, es decir, las estrategias metodológicas en el proceso de enseñanza aprendizaje de la gimnasia para los estudiantes de Educación Física de la Escuela Profesional de Educación de la Universidad Nacional De San Antonio Abad Del Cusco, 2018 son eficientes.

\section{Discusión}

Finalmente, el proceso enseñanza y aprendizaje de los contenidos se van aplicando progresivamente de acuerdo al ritmo de aprendizaje del estudiante dentro de cada actividad, es decir, el rol del entrenador es de guía, orientación y ayuda en dicho proceso de la mejor manera para lograr los objetivos y metas planteadas. Además, se aplican estrategias metodológicas de acuerdo a cada necesidad del atleta para mejorar el rendimiento competitivo (Pinela, 2016).

Por su parte, se debe fomentar la revisión de estudios para elevar el conocimiento pedagógico (Mina y Andino, 2017). Esto se corrobora con la investigación realizada donde el $71 \%$ de los profesores encuestados están "Totalmente de acuerdo" en que debería formularse trabajos, con los estudiantes de la especialidad de Educación
Física según requerimientos del MINEDU, así como también el $42 \%$ de los estudiantes encuestados están "De acuerdo" en que se debería formularse trabajos, con los estudiantes de la especialidad de Educación Física. También, el $100 \%$ de los profesores encuestados están "Totalmente de acuerdo" en que se debe incidir con cursos, seminarios y conferencias sobre las estrategias metodológicas en la enseñanza de la gimnasia.

Además, cuando no se utiliza un método de enseñanza óptimo generando como consecuencia la pérdida de los objetivos planificados (Ticona, 2017), es decir, carece de conocimientos teóricosprácticos, que les permita comprender el tema para un mejor tratamiento durante las sesiones de entrenamiento, dificulta el proceso de enseñanzaaprendizaje de la gimnasia (Estupiñán y Pérez, 2015).

En efecto, la organización y el tratamiento adecuado del contenido de aprendizaje, a su vez, el uso de estrategias metodológicas adecuadas y variadas permiten mejorar las destrezas gimnásticas (Hinostroza, 2019), tal como lo comprueba el $72 \%$ de los profesores y el $46 \%$ de los estudiantes encuestados.

Por su parte, no se considera la importancia que tiene la gimnasia como recurso pedagógico y disciplina en la Educación Física. Por último, se recomienda incentivar competencias gimnásticas para motivar a los jóvenes de las instituciones educativas a participar activamente en dicha disciplina (Huamani, 2019).

\section{CONCLUSIONES}

Las estrategias metodológicas en el proceso de enseñanza y aprendizaje de la gimnasia para los estudiantes de Educación Física de la Escuela 
Profesional de Educación de la Universidad Nacional de San Antonio Abad Del Cusco para el año 2018, evidencian el resultado de la prueba de hipótesis a través de la media aritmética que es el 2,57. Esto significa que dichas estrategias metodológicas aplicadas en el proceso de enseñanza-aprendizaje de la UNSAAC, son eficientes.

Tanto el 53\% de los docentes y el $40 \%$ de los estudiantes están totalmente deacuerdoy deacuerdo, respectivamente, con las metodologías aplicadas en la enseñanza de la Educación Física, por lo que consideran que las informaciones de los diversos temas que representan esas asignaturas cumplen y satisfacen las necesidades de los involucrados por lo que los conocimientos generados son eficientes, permitiendo que el desarrollo integral de los estudiantes sea de calidad.

\section{REFERENCIAS}

Barragán, D. (2018). Orientaciones Básicas Para Mejorar El Proceso De Enseñanza-Aprendizaje De La Gimnasia A Manos Libre En Estudiantes De 6-7 Años. [Tesis de pregrado, Universidad de Guayaquil, Ecuador]. http://repositorio. ug.edu.ec/bitstrea m/redug/34740/1/ Barrag\%c3\%a1n\%20\%20Mieles\%20David\%20 San\%20116-2018.pdf

Cachorro, G. y Giles, M. (2019). Sistematización de Experiencias en Educación Física. Editorial de la Universidad de La Plaza.

Escuela de Gimnasia. (2016). Club Escuela de Gimnasia de Lima. http://www.gimnasia.pe/

Estupiñán, M. y Pérez, M. (2015). Indicaciones Metodológicas Para La Percepción De Tiempo Y Ritmo En La Gimnasia Rítmica, Categoría Pioneril. DeporVida, 12 (24). 12-26.

Guzmán, J. (2019). La Educación Física como acceso a la formación de valores en los estudiantes del quinto grado de educación primaria de la I.E.
Mixto No 50028 Occhullo Grande "Sagrado Corazón de Jesús" Cusco - 2018. [Tesis de maestría, Universidad Nacional San Agustín de Arequipa, Perú]. http://bibliotecas.unsa.edu. pe/handle/UNSA/9932

Hinostroza, L. (2019). Planeación en la enseñanza de la Educación Física para el éxito del proceso de enseñanza-aprendizaje. [Tesis de pregrado, Universidad Nacional de Tumbes, Perú]. http://repositorio.untumbes.edu.pe/handle/ UNITUMBES/1594

Huamani, L. (2019). La gimnasia rítmica en el desarrollo psicomotor de los estudiantes de las instituciones educativas secundarias. [Tesis de pregrado, Universidad Nacional de Tumbes, Perú]. http://repositorio.untumbes.edu.pe/ handle/UNITUMBES/1268

Mina, A., y Andino, M. (2017). Aspectos metodológicos en el proceso de enseñanzaaprendizaje de la gimnasia en estudiantes de Educación Física. Ecos de la Academia, 6 (3). 133-144.

Pinela, W. (2016). Evaluación de una metodología inclusiva desde la Ciencia de la Cultura Física y el deporte para personas con discapacidad en la disciplina de Gimnasia Deportiva del proyecto "Deporte por Sonrisa" de la FEDER [Tesis de pregrado, Universidad de Guayaquil, Ecuador]. http://repositorio.ug.edu.ec/bitstream/ redug/26683/1/Pinela $\% 20$ Rodriguez $\% 20$ Wendy\%20Liliana\%20\%20236-2016.pdf

Reguena, X. (2018). Diseño y evaluación de un programa de Gimnasia Acrobática para la mejora delashabilidadessocialesypersonaleseneducación secundaria. [Tesis Doctoral, Universidad de Vigo, España]. http://www.investigo.biblioteca. uvigo.es/xmlui/bitstream/handle/11093/1018/ dise \%c $3 \%$ b $1 o_{-}$evaluaci $\%$ c $3 \%$ b $3 n_{-}$ programa_gimnasia_acrob\%c3\%a1tica. pdf? sequence $=1$ \&isAllowed $=y$

Sánchez, M. (2019). Gimnasia sueca y desarrollo motor grueso en niños de 5 años del jardín 
"Despertar" Trujillo. [Tesis de pregrado, Universidad Nacional de Trujillo, Perú]. https:// revistas.unitru.edu.pe/index.php/SCIENDO/ article/view/2405

Ticona, W. (2017). Factores que influyen en la enseñanza aprendizaje de la gimnasia deportiva en las Instituciones Educativas primarias de la Ciudad de Puno - 2015. [Tesis de pregrado, Universidad Nacional del Altiplano, Perú]. http://repositorio.unap.edu.pe/handle/ UNAP/4207

UNSAAC (2017). Plan de Estudios de la Facultad de Educación y Ciencias de la Comunicación. http:// eu.unsaac.edu.pe/home/
Villalobos, P. (2015). Creación Del Coliseo Multideportivo Municipal Para Desarrollar Las Capacidades Deportivas Del Distrito De Echarati Provincia De La Convención - Cusco. [Tesis de pregrado, Universidad Nacional del Centro del Perú, Perú]. http://181.65.200.104/bitstream/ handle/UNCP/4748/Villalobos\%20Torres. pdf? sequence $=1 \&$ isAllowed $=y$ 